\title{
The chemical potential of light in fluorescent solar collectors
}

\author{
T. J. J. Meyer ${ }^{\mathrm{a})}$ and T. Markvart \\ Solar Energy Laboratory, Materials Research Group, School of Engineering Sciences, \\ University of Southampton, Highfield, Southampton SO17 1BJ, United Kingdom
}

(Received 1 November 2008; accepted 8 February 2009; published online 25 March 2009)

\begin{abstract}
The energetic aspect, discussed by the means of the chemical potential, involved into the absorption and radiation processes occurring in the operation of fluorescent solar collectors is of interest in this publication. The chemical potential of the fluorescent light incident on the solar cell is characterized by studying the fluorescence spectrum emitted by a special type of fluorescent collector, where absorption and fluorescence take place in a liquid medium, in effect a liquid fluorescent collector. It is shown that photon reabsorption (known also as photon recycling) gradually brings the emitted photon flux into thermal equilibrium with the collector. The fluorescence photon distribution is then characterized by a specific temperature, obtained from the Kennard-Stepanov law, and a chemical potential given by the generalized Planck's law. We find that the chemical potential of the fluorescent light incident on the solar cell is nearly equal to the thermodynamical limits imposed by a detailed balance argument. This equality indicates that nonradiative losses do not affect the chemical potential of the light escaping from the collector, a major benefit in comparison to simple semiconductor based solar cells where nonradiative losses significantly affect the open circuit voltage. (C) 2009 American Institute of Physics. [DOI: 10.1063/1.3097328]
\end{abstract}

\section{INTRODUCTION}

Fluorescent solar collectors (FSCs) represent an attractive photovoltaic technology, with the potential to decrease substantially the size of solar cells. In conventional FSCs, the collector generally contains fluorescent dyes embedded in a transparent medium such as polymethyl methacrylate, polycarbonate, glass, or even a liquid solution (Fig. 1). When illuminated, the dyes absorb a part of the incoming light and re-emit photons inside the fluorescent layer at a longer wavelength. ${ }^{1}$ A large part of the re-emitted photon flux is trapped by total internal reflection and collected by the solar cell. For example, with a typical refractive index of materials used in the fabrication of fluorescent collectors $(n \sim 1.5)$, the probability that a photon becomes trapped after each emission equals 0.75 . Below, this probability is denoted by $1-P$.

The thermodynamic limitations for the efficiency of FSCs have been recently discussed in a series of publications. $^{2-6}$ The key in this argument is the assumption of thermal equilibrium for the emitted photons, resulting in an equal chemical potential $\mu$ for the trapped photon flux. ${ }^{2,4,6}$ In this paper, the chemical potential of the edge fluorescent flux $\left(\mu_{\text {flux }}\right)$, the fluorescent flux incident on the solar cell, is characterized and compared with the chemical potential of the excited state of the dye $\left(\mu_{\text {dye }}\right)$. We verify that these chemical potentials are indeed identical and, for practical purposes, $\mu_{\text {dye }}$ equals the chemical potential of an ideal photon gas.

The supporting experimental results were obtained with the help of fluorescent collectors based on a thin glass cuvette, which contains a fluorescent solution: in effect, a liquid fluorescent collector. This collector has the advantages of

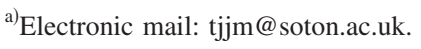

homogeneity and easy replacement of the matrix. The use of liquid fluorescent solution also facilitates the application of the Kennard-Stepanov ${ }^{7}$ relation, which is needed to characterize the thermal equilibrium of the photon flux.

The structure of this paper is as follows: Sections II-IV present the different approaches employed to characterize the three chemical potentials $\left(\mu_{\text {flux }}, \mu_{\text {dye }}\right.$, and $\left.\mu_{\text {theo }}\right)$, Sec. $V$ gives a brief discussion of the effective temperature of fluorescent radiation, Sec. VI describes the experimental setup, and Sec. VII presents the results and discussion.

Before embarking on a detailed analysis, a brief summary of the essential concepts is in place.

- The chemical potential of the edge fluorescent flux $\left(\mu_{\text {flux }}\right)$ relies on the hypothesis that this flux reaches a

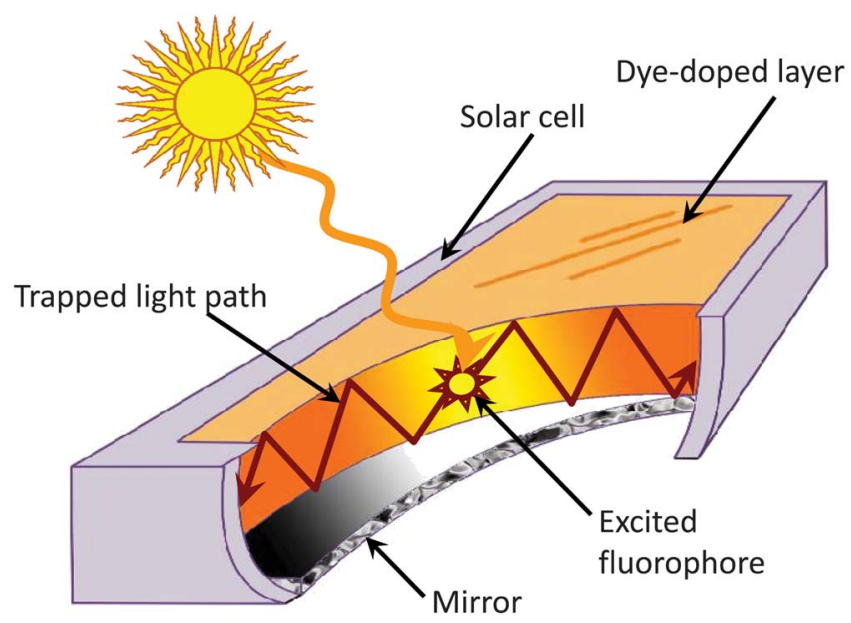

FIG. 1. (Color online) Cut away of a FSC, showing the large area $A_{\text {front }}$ of the fluorescent medium (orange) exposed to sunlight and the solar cells (gray) with an area $A_{\text {edge }}$ placed at the edge of the collector. The optical gain $G$ of these devices is equal to the ratio $A_{\text {front }} / A_{\text {edge. }}$. 


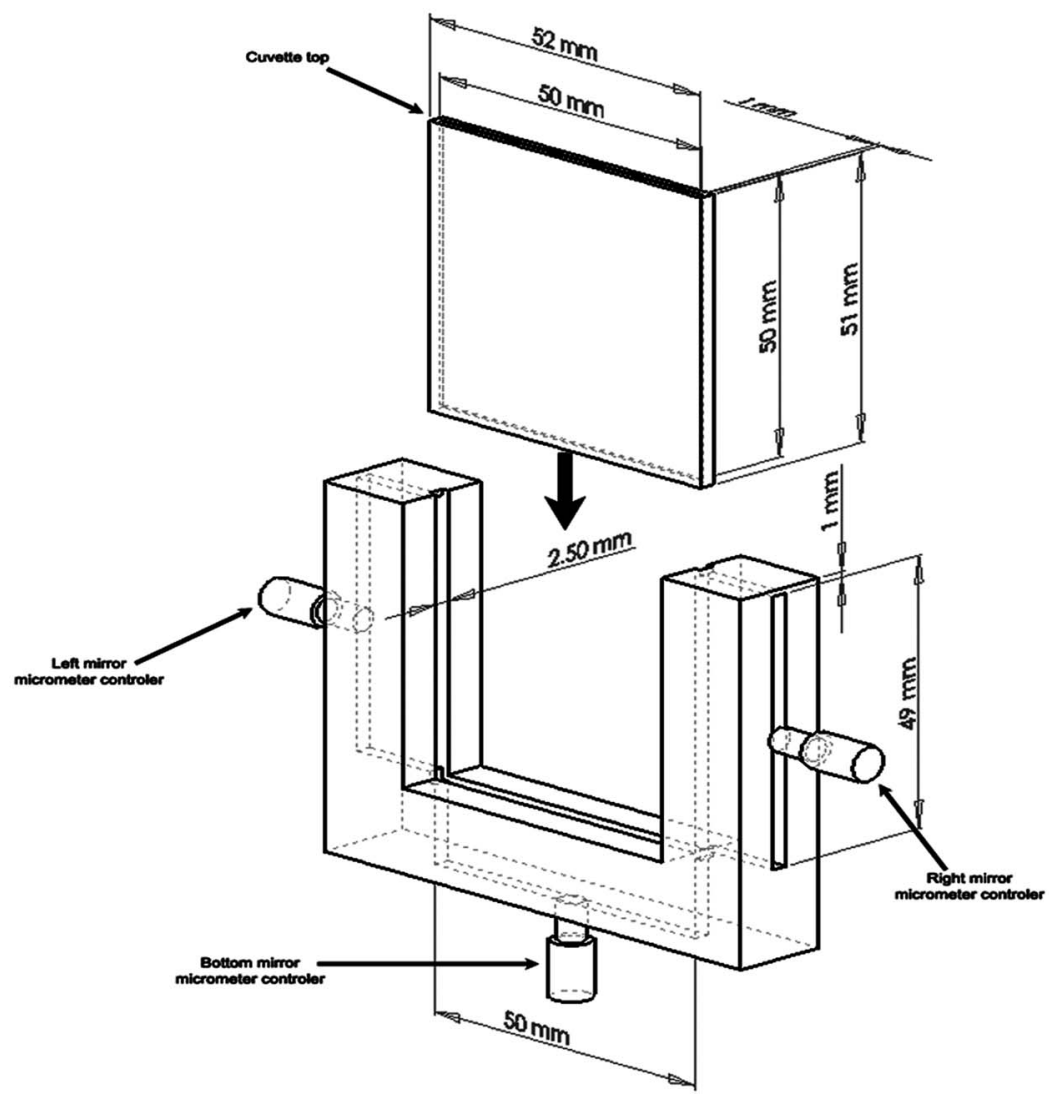

FIG. 2. Perspective view of the cuvette and sample holder showing how the cuvette was slotted in the holder.

thermal equilibrium upon multiple reabsorption and re-emission events. The photon distribution is consequently described by a specific temperature and a chemical potential given by a generalized Planck's law $^{8,9}$ (Sec. II).

- The effective chemical potential of the photoexcited dye $\left(\mu_{\text {dye }}\right)$ is defined here as in standard thermodynamic texts (see, e.g., Ref. 10) (Sec. III).

- A theoretical estimate of the photon chemical potential $\left(\mu_{\text {theo }}\right)$ is determined by a detailed balance argument between the incident and emitted radiations. This expression resembles the Carnot engine type relation, ${ }^{11}$ formerly used with success for the open circuit voltage of solar cells, which has been adapted to the specific characteristics of fluorescent collectors ${ }^{4}$ (Sec. IV).

\section{PHOTONS AT EQUILIBRIUM WITH MATTER}

Let us assume that the quantum yield $\phi$ of the dye is unity. To a good approximation, this is true for all the dyes studied in this paper. Such dye re-emits all the absorbed photons within the collector. As previously discussed in Refs. 4, 6 , and 12 this perfect emission-absorption cycle (also known as photon recycling) ${ }^{13}$ brings the re-emitted light into thermal equilibrium with the absorbing/fluorescent species. The photon occupation numbers can then be described by the Bose-Einstein distribution with, in general, a nonzero chemical potential $\mu$ as follows:

$$
P_{\text {stat }}(T, \mu, \nu)=\frac{1}{\exp \left(\frac{h \nu-\mu}{k T}\right)-1},
$$

where $k$ is the Boltzmann constant, $h$ is the Planck constant, and $T$ is the temperature. Based on this argument one expects that the temperature, $T$, is equal to the temperature of the dye. As we shall see in Sec. V, a more rigorous determination of the temperature which enters the Kennard-Stepanov relation $^{7}$ is, in many cases, needed. The temperature that results will be denoted by $T^{*}$. Noting that the exponential term in the numerator of Eq. (1) is large, the fluorescent spectra emitted from the edge of the collector can thus be approximated by

$$
B\left(T^{*}, \mu_{\text {flux }}, \nu\right) \approx \frac{2 \pi h \nu^{3}}{(c)^{2}} \exp \left(\frac{-h \nu}{k T^{*}}\right) \exp \left(\frac{\mu_{\text {flux }}}{k T^{*}}\right),
$$

where $c$ is the speed of light, and we inserted the chemical potential of the luminescent flux $\mu_{\text {flux }}$.

\section{CHEMICAL POTENTIAL OF THE DYE}

Let us consider a simple photochemical reaction where a molecule $N$ in the ground state absorbs a photon of energy $h \nu_{0}$ and becomes excited $\left(N^{*}\right)$. Under steady illumination, the chemical potential of the excited dye can be written as ${ }^{10}$

$$
\mu_{\mathrm{dye}}=h \nu_{0}+k T^{*} \ln \left(\frac{\left[N^{*}\right]}{[N]}\right),
$$

where $\left[N^{*}\right]$ and $[N]$ represent the concentration of the excited molecules and the concentration of molecules in the ground 

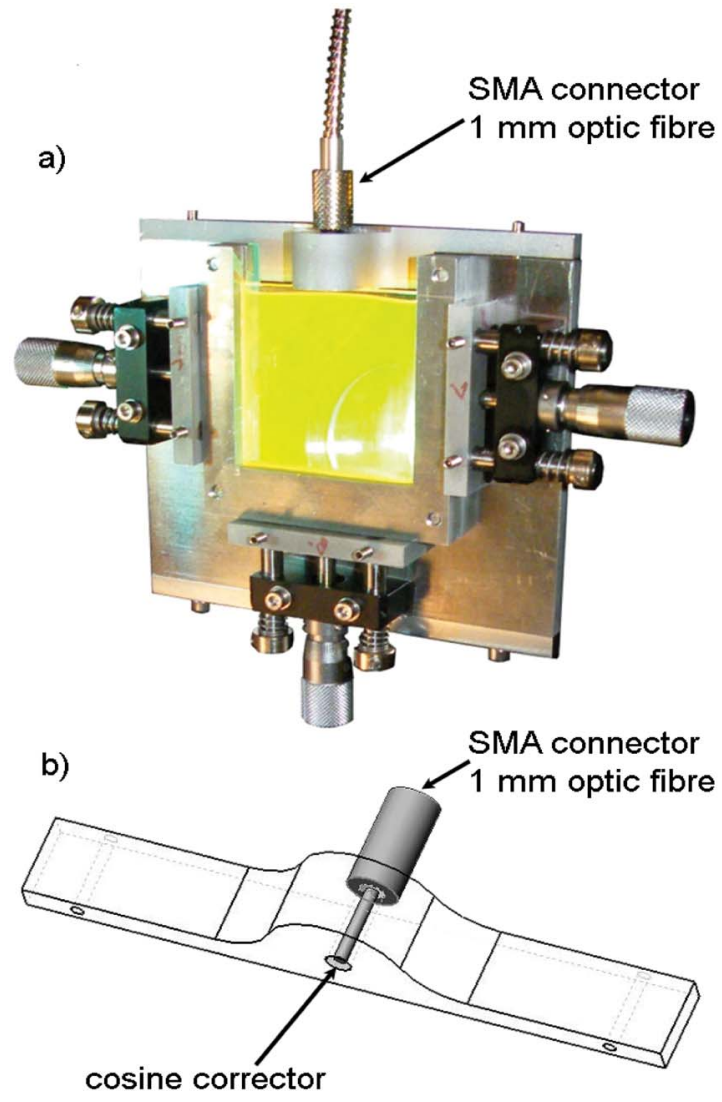

FIG. 3. (Color online) (a) Actual picture of the sample holder with a cuvette in operation; here the mounted top bracket was designed to measure the edge fluorescence spectrum using a cosine-corrected optical fiber. (b) Diagram of the metallic bracket holding the cosine-corrected fiber.

state, respectively. Expression (3) can be interpreted as the difference between the chemical potentials of the ground and excited states of the dye molecule-in other words, the "splitting" of the two chemical potentials, as commonly used in quantum solar energy conversion. For a simple model where the vibrational potential curves are approximated by parabolas with equal frequencies, the energy separation represented by $h \nu_{0}$ corresponds to the midpoint between the wavelength of maximum absorption and maximum fluorescence. ${ }^{14}$

Neglecting reabsorption in the escape cone, in a fluorescent collector, the concentration $\left[N^{*}\right]$ of the excited dye molecules can be approximated by ${ }^{6}$

$$
\left[N^{*}\right]=\frac{\dot{N}_{\mathrm{abs}} \tau_{\mathrm{tot}}}{1-\phi(1-P) R},
$$

where $\tau_{\text {tot }}$ is the total de-excitation lifetime of the dye, the absorbed photon flux $\dot{N}_{\text {abs }}$ is obtained using the absorption coefficient of the dye and Beer-Lambert's law, the photon reabsorption probability $R$ in the collector can be determined by the methods developed in Ref. 6 , and the probability $P$ of emission into the critical angle is given by

$$
P=1-\sqrt{1-1 / n^{2}},
$$

where $n$ is the refractive index of the collector. In the case of inhomogeneous collectors, which contain several parts with

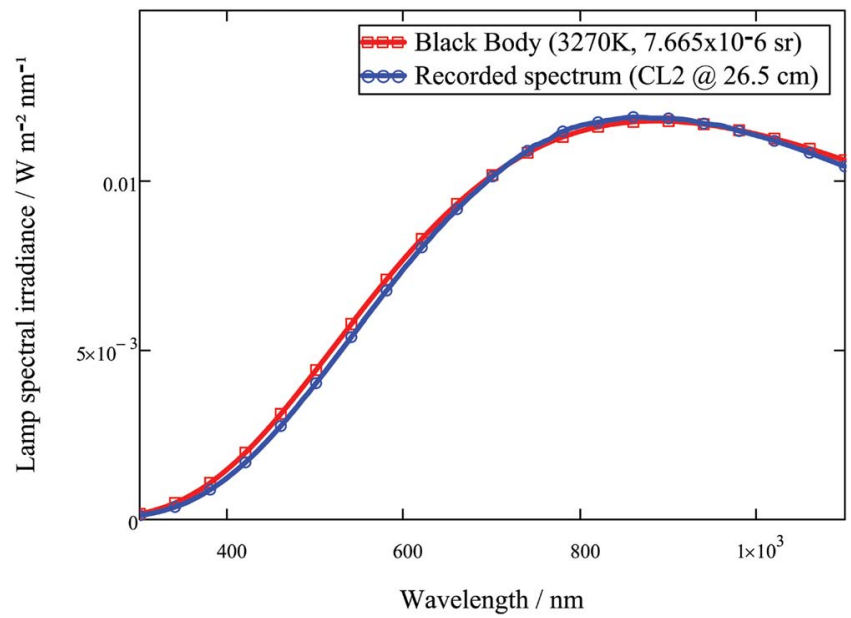

FIG. 4. (Color online) Comparison between a black body (plain line) at temperature $T=3270 \mathrm{~K}$ and solid angle $\Omega_{\text {sun }}=7.665 \times 10^{-6} \mathrm{sr}$, and the spectrum of the CL2 lamp (dotted line) measured $26.5 \mathrm{~cm}$ away from the tungsten filament.

different refractive indices, the re-emission probability $P$ was determined by ray-tracing techniques (see Sec. VI B).

\section{COMPARISON WITH THEORY}

It is of interest to compare the experimental values of $\mu_{\text {flux }}$ and $\mu_{\text {dye }}$ with theory. To this end we shall use the upper limit of the chemical potential, which has been obtained in Ref. 4. Considering a collector illuminated by a thermal source at $T_{s}$ under a solid angle $\Omega_{s}$ the chemical potential of the photons escaping the device is given by

$$
\begin{aligned}
\mu_{\text {theo }}= & h \nu_{\text {abs }}\left(1-\frac{T_{c}}{T_{s}}\right)+k T_{c} \ln \left(\frac{T_{s}}{T_{c}}\right)+k T_{c} \ln \left(\frac{\Omega_{s}}{\pi}\right) \\
& +k T_{c} \ln \left[\frac{1+\wp\left(\nu_{\mathrm{abs}}, T_{c}\right)}{1+\wp\left(\nu_{\mathrm{em}}, T_{c}\right)}\right]+k T_{c} \ln (1-Q c),
\end{aligned}
$$

where $\nu_{\mathrm{em}}$ is the fluorescence emission frequency, $\nu_{\mathrm{abs}}$ is the absorption frequency, $Q c$ is the collection efficiency (probability of photon emission at the edge of the collector, as defined in Refs. 1 and 6), $T_{c}$ is the temperature of the collector, and the function $\wp(\nu, T)$ is a small correction term equal to

$$
\wp(\nu, T)=2 \frac{k T}{h \nu}+2\left(\frac{k T}{h \nu}\right)^{2} .
$$

Equation (6) assumes that the chemical potential of the incident light is zero and, as before, neglects stimulated emission. This Carnot engine type relation resembles the expression of Ruppel and Würfel ${ }^{11}$ who used the first three terms to determine the open circuit voltage of a solar cell. The addition of the last two terms gives a more accurate formula for the operation of FSCs, taking into account the difference between the absorption and emission wavelengths and the reduction in the photon chemical potential on account of the collection efficiency.

The solid angle and the temperature of the source were determined by comparing the illumination spectrum with a black body radiator as shown in Fig. 4. The collection effi- 

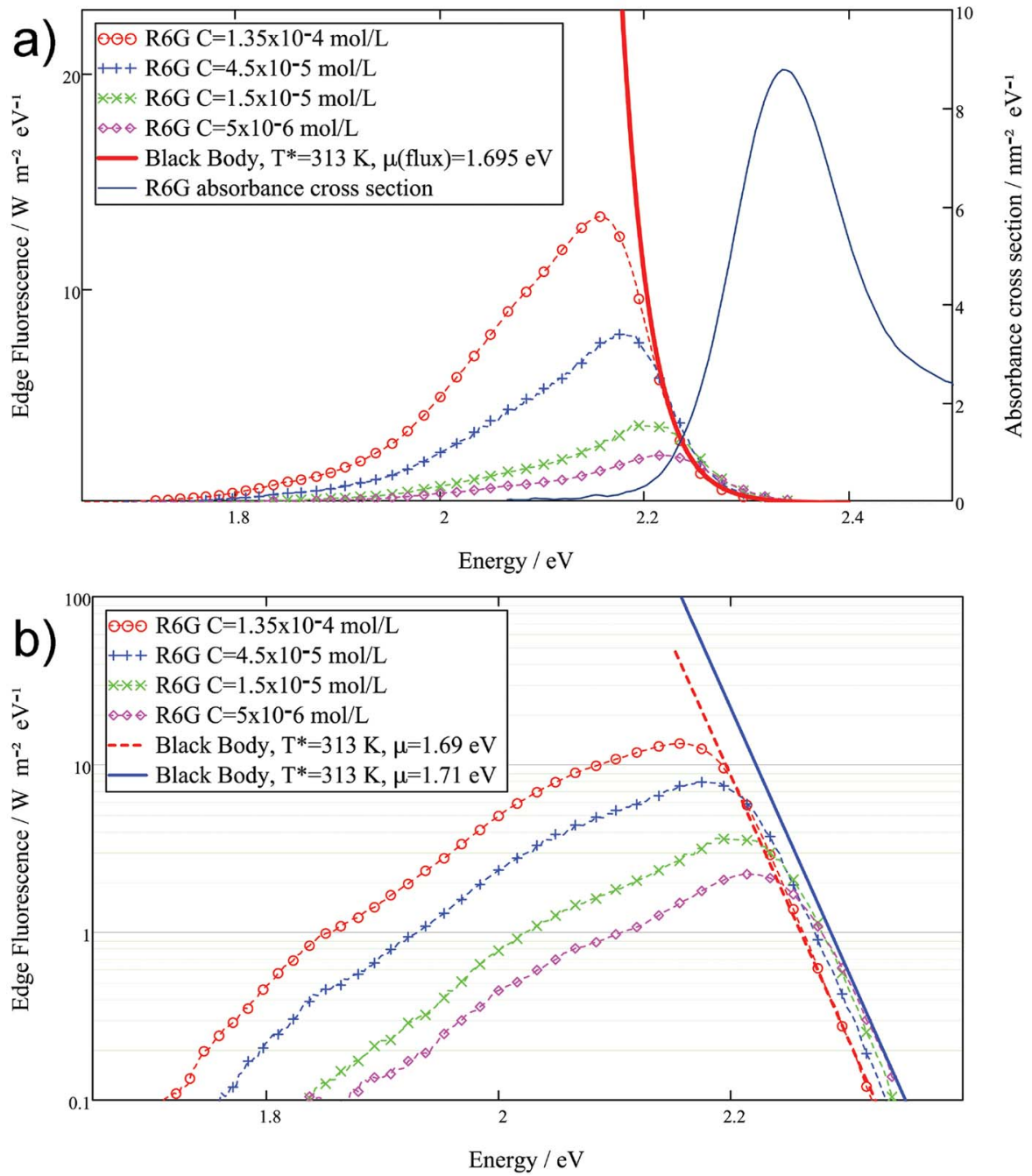

FIG. 5. (Color online) (a) Typical edge fluorescent spectra of R6G compared with a black body function at $T^{*}=313 \mp 8 \mathrm{~K}$ and $\mu_{\text {flux }}$ $=1.695 \pm 0.01 \mathrm{eV}$. (b) Log plot of (a) showing the uncertainty band on the chemical potential calculated. The numbers in the legend give the dye concentration in the solvent. ciency was measured directly as the ratio of the number of photons emitted at the edge over the number of photons absorbed.

\section{THE KENNARD-STEPANOV THEORY}

The absorption or fluorescence by a dye molecule involves energy redistribution between the various vibrational energy levels and the solvent. This redistribution of energy is extremely rapid - in the order of a picosecond, which is considerably shorter than the lifetime of the excited state. It is usually assumed that a thermal equilibrium between the excited state of the dye and its surroundings is established within this short time period. In a mathematical form ${ }^{7,15}$ one can then say that the absorption cross section $\sigma(\nu)$ and the fluorescent emissive power $I(\nu)$ of a molecule at temperature $T$ are related by the Kennard-Stepanov relation, which is generally written in the form

$$
\ln \left[\frac{c^{2} I(\nu)}{8 \pi h \nu^{3} \sigma(\nu)}\right]=-\frac{h \nu}{k T}+D(T),
$$

where $D(T)$ is a constant, which depends only on the temperature.

It has been observed experimentally ${ }^{16}$ that Eq. (8) often holds, although the temperature $T$ is not equal to the tem- perature of the emitting substance. As highlighted in Sec. II, we adopt the notation $T^{*}$ for the effective temperature to be used for characterizing the relationship between the absorption and emission processes.

\section{EXPERIMENTAL DETAILS}

\section{A. Liquid collectors}

The liquid collector used in this work is similar to a "stretched" spectroscopic cuvette; a quartz cell of optical dimensions $50 \times 50 \mathrm{~mm}^{2}$ with a spacing of $0.5 \mathrm{~mm}$ was filled with a solvent. This quartz cuvette $\left(\text { Suprasil }^{\circledR} n=1.46\right)^{17}$ was fabricated by Hellma, Germany (see Fig. 2). Each quartz wall is $1 \mathrm{~mm}$ thick, leading to an optical gain of 20. All structural parts were polished to extreme flatness and formed a homogeneous structure produced via a direct fuse process. A silicon solar cell was then coupled to one edge of the cuvette, creating a working device (Fig. 3).

\section{B. Sample preparation}

The spectroscopic studies were carried out using Rhodamine 6G (R6G) and Rhodamine 101 (R101) (both from Acros, $99.5 \%$ purity), with properties well documented 

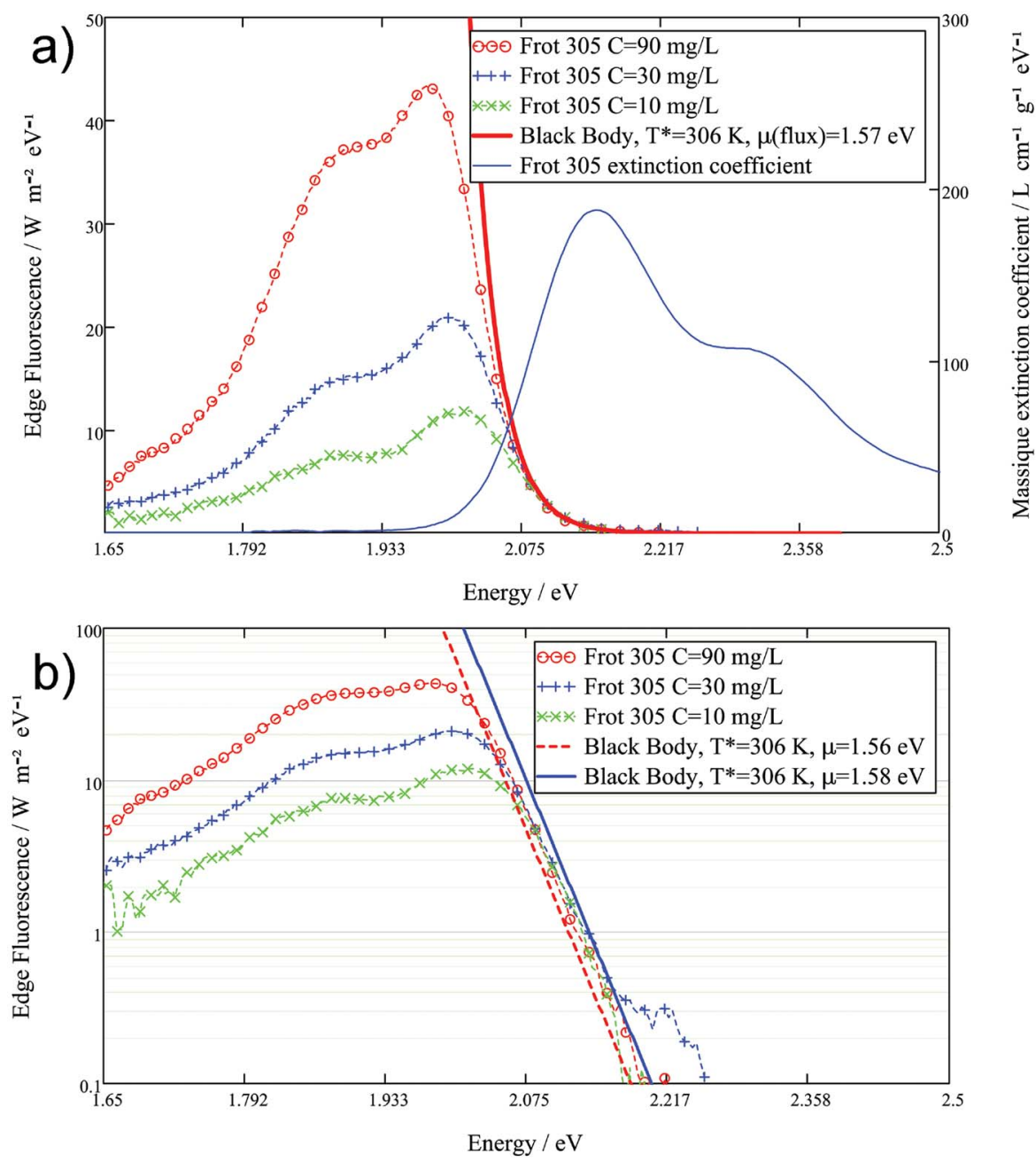

FIG. 6. (Color online) (a) Typical edge fluorescent spectra of F305 compared with a black body function at $T^{*}=306 \mp 8 \mathrm{~K}$ and $\mu_{\text {flux }}$ $=1.57 \pm 0.01 \mathrm{eV}$. (b) Log plot of (a). The numbers in the legend give the dye concentration in the solvent. in literature. ${ }^{18,19}$ We also used a new lumogen dye from BASF [Frot 305 (F305)]. Stock solutions ranging from $5 \times 10^{-6}$ up to $1.35 \times 10^{-4} \mathrm{~mol} / \mathrm{L}$ were prepared for the rhodamine dyes. A stock solution from 10 up to $90 \mathrm{mg} / \mathrm{L}$ was prepared for the F305 dye (these concentrations are reported in $\mathrm{mg} / \mathrm{L}$ since the molar mass of lumogen dyes is unknown). Each of the solutions was independently made from a weighted mass (Metler Toledo XP56, $1 \mu \mathrm{g}$ readability) of crystalline dye and dissolved in a known volume of spectroscopic quality of solvent. Dichloromethane (DCM) was the solvent used for studying the F305 dye $(\phi=0.98) ;{ }^{20}$ DCM has a refractive index of $1.42,{ }^{21}$ close to the Suprasil quartz, avoiding optical mismatch at the quartz/liquid interface. During the experiment, measures were taken to control the temperature of the solution to prevent evaporation. Ethanol was used as a solvent for the rhodamine dyes. The latter has a refractive index of $1.36,{ }^{21}$ creating an optical mismatch at the solvent/cuvette interface and the need for a more complex model for the photon transport in the collector. This model was developed by ray tracing using commercial software (TracePro ${ }^{\circledR}$ ), yielding an effective value of $P$, which was now used in place of Eq. (5). Details of this model will be published in a separate article; ${ }^{22}$ suffice it to say that the chemical potential is insensitive to the precise details, and the mismatch results in an uncertainty of $\mp 0.01 \mathrm{eV}$.

\section{Sample holder and absolute spectroscopic measurements}

The aluminum sample holder (Figs. 2 and 3) contains three extrusions, which allow micrometer-controlled mirrors, to slide through the sample holder. With the presence of mirrors, the structure resembles the infinite strip geometry of Weber and Lambe. ${ }^{1}$ The mirrors used in this publication were $\frac{1}{4}$ wavelength first surface mirrors from Edmund optic with a reflectivity $>90 \%$ across the visible spectrum. A back mirror (dimension $50 \times 50 \mathrm{~mm}^{2}$, not shown in Figs. 2 and 3) was also used during the fluorescent measurement. During the experiments the collector was mounted upright, and the edge fluorescence was detected from the open (top) edge of the cuvette.

A calibrated Avantes spectrometer AvaSpec-2048, grating UA (200-1000 nm), slits $25 \mu \mathrm{m}$, based on the symmetrical Czerny-Turner design with a 2048 pixel charge-coupled device $(\mathrm{CCD})$ detector array was used for all absorbance and fluorescence measurements. A Bentham CL2 universal spectral irradiance standard lamp (calibration traceable to NPL Teddington, UK) with a wavelength range $250-3000 \mathrm{~nm}$ and a color temperature of $3270 \mathrm{~K}$ (Fig. 4) was used to illuminate the liquid collector. The distance between the lamp and the collector was $26.5 \mathrm{~cm}$ and all the measurements took place in a light proof black box with fans to maintain a temperature 

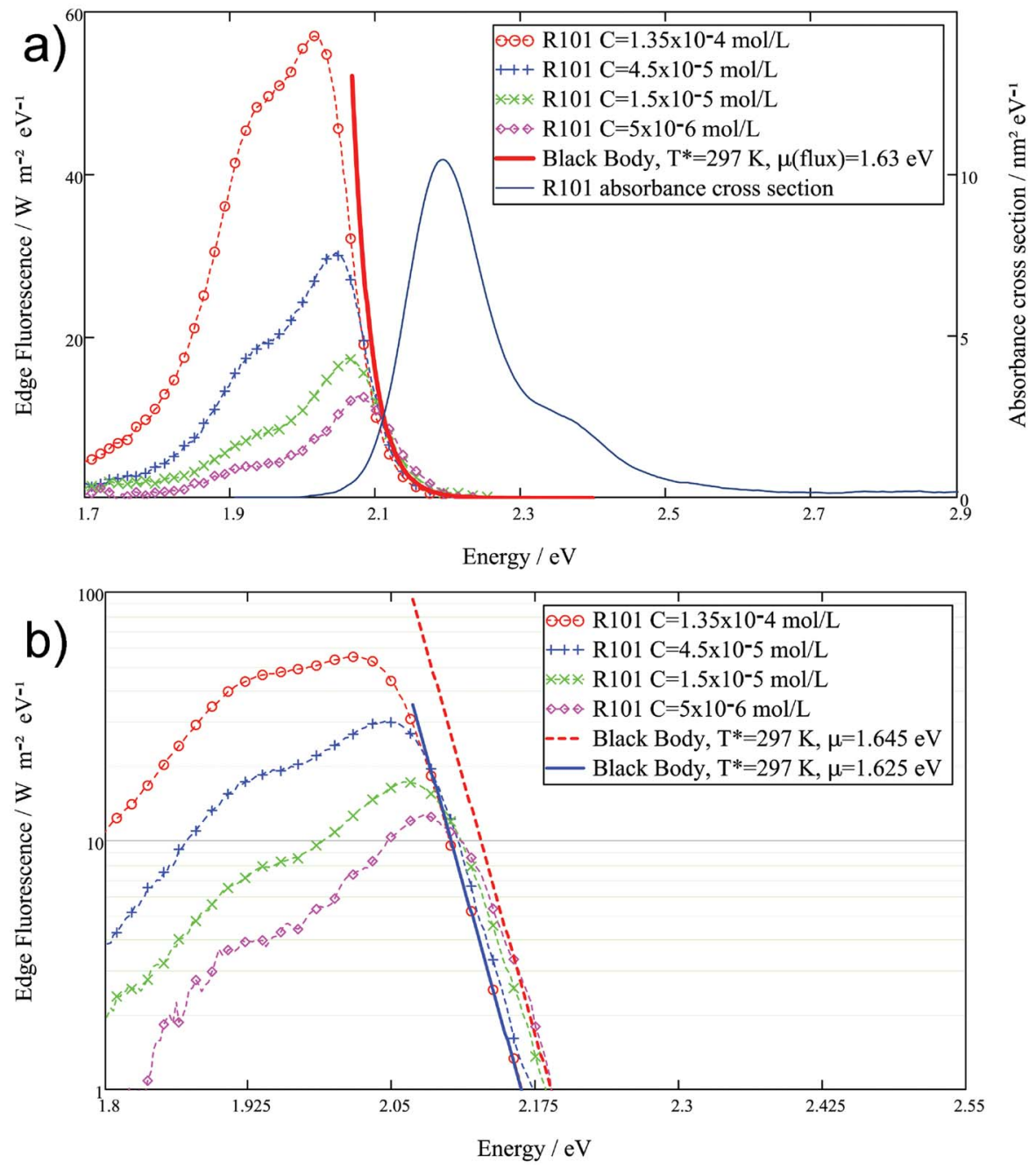

FIG. 7. (Color online) (a) Typical edge fluorescent spectra of R101 compared with a black body function at $T^{*}=297 \mp 8 \mathrm{~K}$ and $\mu_{\text {flux }}$ $=1.635 \pm 0.01 \mathrm{eV}$. (b) $\log$ plot of (a). The numbers in the legend give the dye concentration in the solvent.

between 20 and $25^{\circ} \mathrm{C}$ during the experiments. The absorbance spectra were measured in a standard fashion, with an optical fiber placed at the back in the center of the collector, integration time of $2 \mathrm{~ms}$, and averaged 100 times.

The edge fluorescence setup for the liquid was a $90^{\circ}$ angle detection of fluorescence. The measurement procedure for absolute characterization of the edge fluorescent photon flux was a two step process.

(1) The edge fluorescence spectra were measured using the Avantes and a $1 \mathrm{~mm}$ diameter cosine-corrected optical fiber placed in the middle of the edge of the collector as shown in Fig. 3. Cosine-corrected fiber optic turns into irradiance probes collecting radiation over $2 \pi \mathrm{sr}$, eliminating optical interface problems associated with the light collection sampling geometry inherent to bare fibers and other sampling devices. The diffusing material used in the cosine corrector is a thin disk of Teflon $3900 \mu \mathrm{m}$ diameter optimized for applications from 200 to $1100 \mathrm{~nm}$ that sits at the end of a stainless steel barrel screwed on the fiber SubMiniature version A (SMA) connector as shown in Fig. 3(b). The spectra were recorded with an integration time of $300 \mathrm{~ms}$, averaged 50 times with their maximum intensity normalized to unity. Only the corrected profile of fluorescence is of interest at this stage.
(2) Once the contour of the fluorescence spectrum was recorded, the optic fiber was removed and replaced with a calibrated solar cell covering the whole top side of the cuvette. The cell and the collector were optically coupled using an index matching gel (ThorLabs). The short circuit $\left(I_{\mathrm{sc}}\right)$ current recorded by the cell was used to scale in intensity the previously recorded fluorescence spectra. The normalized fluorescence profile was multiplied with an appropriate scaling constant to obtain

$I_{\mathrm{sc}}=\int \operatorname{SR}(\lambda) F_{\text {edge }}(\lambda) d(\lambda)$,

where $\operatorname{SR}(\lambda)$ is the spectral response of the cell and $F_{\text {edge }}(\lambda)$ is the scaled fluorescence spectrum.

The short circuit current of the solar cell was measured with a calibrated analog to digital dc amplifier of Bentham 487 spectrometer. The spectral response of the solar cell was characterized in a solar cell simulator (T.S. Space Systems) equipped with a xenon lamp and a filter selection wheel (400-1100 nm, step of $50 \mathrm{~nm}$ ) against a national renewable energy laboratory (NREL) calibrated solar cell. The spectral response data were checked against the Bentham spectral response system. 
TABLE I. Summary of the results, showing the different chemical potentials characterized for the dyes R101, R6G, and F305. The uncertainty related to the chemical potentials given in this table was estimated to be 0.01 $\mathrm{eV}$; other uncertainties are directly indicated in the table. The missing data for F305 are due to the lack of information on the molar mass of the dye.

\begin{tabular}{|c|c|c|c|c|c|c|c|c|}
\hline Dye & Solvent & $\begin{array}{l}h v_{0} \\
(\mathrm{eV})\end{array}$ & $\begin{array}{c}\tau_{\text {tot }} \\
\text { (ns) }\end{array}$ & $\phi$ & $\begin{array}{c}T^{*}, T_{\text {col }} \\
(\mathrm{K})\end{array}$ & $\begin{array}{l}\mu_{\text {flux }} \\
(\mathrm{eV})\end{array}$ & $\begin{array}{l}\mu_{\text {dye }} \\
(\mathrm{eV})\end{array}$ & $\begin{array}{l}\mu_{\text {theo }} \\
(\mathrm{eV})\end{array}$ \\
\hline & & & $4.46 \pm 0.03^{b}$ & & $297 \pm 8$ & & & \\
\hline \multirow[t]{2}{*}{ R101 } & Ethanol $n=1.36^{\mathrm{a}}$ & 2.15 & $4.92 \mathrm{~S} \& \mathrm{~B}$ & $1^{\mathrm{b}}$ & 297 & 1.63 & 1.64 & 1.68 \\
\hline & & & $3.99 \pm 0.03^{c}$ & & $313 \pm 8$ & & & \\
\hline \multirow[t]{2}{*}{ R6G } & Ethanol $n=1.36^{\mathrm{a}}$ & 2.29 & $3.88 \mathrm{~S} \& \mathrm{~B}$ & $0.95^{\mathrm{c}}$ & 297 & 1.69 & 1.71 & 1.81 \\
\hline & & & $5.74 \pm 0.02$ & & $306 \pm 8$ & & & \\
\hline F305 & $\operatorname{DCM} n=1.42^{\mathrm{a}}$ & 2.08 & $\cdots$ & $0.98^{\mathrm{d}}$ & 295 & 1.57 & $\cdots$ & 1.64 \\
\hline
\end{tabular}

\section{RESULTS AND DISCUSSION}

Typical edge fluorescence spectra of fluorescent collectors doped with a single dye are shown in Figs. 5-7. Each edge fluorescence spectrum is also plotted on a logarithmic scale in Figs. 5(b), 6(b), and 7(b) to demonstrate the fit between the edge fluorescence spectra and the function (2). For each concentration studied, fluorescent spectra were obtained under identical illuminations; each graph therefore corresponds to the same incident photon flux. Broad bandpass filters were initially used (e.g., $85 \mathrm{~nm}$ bandpass filter centered at $500 \mathrm{~nm}$ for R6G) to prevent noise in the recorded fluorescence contour. It turned out that fluorescence bands were independent of the excitation source providing that the background light was removed. No filters were used for R101 and F305.

As already pointed out in Ref. 6, edge fluorescent graphs display two facets of photon transport in the collector: (i) a high energy spectral region where reabsorption affects the photon transport and brings photons into thermal equilibrium, and (ii) a low energy spectral region where light travels freely in the collector, trapped by total internal reflection.

Figures 5(a), 6(a), and 7(a) illustrate the two photon transport facets: In the high energy region a black body function fits well the rise of the edge fluorescent spectra, which is independent of the dye concentration. This contrasts with the low energy region, characterized by a negligible absorption cross section of the fluorophore where absorption is weak and the fluorescence intensity is proportional to the concentration, and to the number of photons absorbed. ${ }^{6}$

The remarkable thermodynamic behavior of fluorescence is highlighted in the log plots [Figs. 5(b), 6(b), and 7(b)]. As expected from Eq. (2), the edge fluorescence in the reabsorption region now becomes linear with a slope given by the inverse temperature. The edge fluorescence spectra shown in these graphs resemble the spectrum of thermal radiation using the Kennard-Stepanov temperature of the absorbing/emitting species. The uncertainty related to the chemical potential (dotted and bold lines in the graphs) was estimated to be $0.01 \mathrm{eV}$.

A summary of the results comparing the edge fluorescence chemical potential to the dye chemical potential and the theoretical chemical potential is given in Table I. The lifetime reported in the table for the rhodamine dyes R101 and R6G were taken from literature ${ }^{18,19}$ and checked against the Strickler-Berg relation. ${ }^{23}$ In the case of the recently synthesized F305 dye, the lifetime was measured by a fluorescence lifetime spectrometer (FluoTime 200). The quantum yield of F305 was obtained from literature ${ }^{20}$ and verified by the method of Williams et al. ${ }^{24}$

\section{CONCLUSION}

We showed that fluorescence spectra of an optically thick medium can be compared to a quasi-black-body radiation with a nonzero chemical potential (Figs. 5-7). This comparison demonstrates that photons enter into thermal equilibrium upon perfect absorption and re-emission, resulting in an equal chemical potential $\mu$ for all photons in the system, as postulated in Refs. 2, 4, and 6 . The chemical potential of the edge fluorescence flux, characterized using thermodynamics theory of an ideal photon gas, was shown to be remarkably close to the dye chemical potential and the theoretical limits (Table I). Theoretically, the measured chemical potential can be related to the maximum open circuit voltage $V_{\text {oc }}$ of the edge solar cell since once the photon flux is cooled down, there are no fundamental losses that impede the photoconversion. Hence

$$
q V_{\mathrm{oc}}=\mu_{\mathrm{flux}} \approx \mu_{\mathrm{dye}},
$$

where $q$ is the elementary charge. Equation (10) simply equates the splitting of the two chemical potentials characterizing the photoexcited dye molecule, to the open circuit voltage. This is a similar argument commonly used for solar cells where the splitting of the quasi-Fermi levels defines the upper limit of the open circuit voltage. ${ }^{25}$ The key difference is that the chemical potential $\mu_{\text {flux }}$ in FSCs is closer to the thermodynamical limit than the chemical potential observed for solar cells.

\section{ACKNOWLEDGMENTS}

It is my pleasure to acknowledge many stimulating discussions with my colleagues and friends F. Pace, P. Wright, B. C. Rowan, R. Greef, and C. Hickey on the subject of this paper. A special thanks to L. Danos for measuring the lifetime of the dyes. I would also like to thank the individuals from the industry cooperating with us, especially, B. Calt 
(TracePro) for a free license, P. Bailey (Hellma) helping with the collector design, L. Lyons (Bentham) for his help with the system calibration, and D. Turner (BASF) for kindly donating the lumogen dyes.

${ }^{1}$ W. H. Weber and J. Lambe, Appl. Opt. 15, 2299 (1976).

${ }^{2}$ T. Markvart, L. Danos, P. Kittidachachan, and R. Greef, Detailed balance efficiency of ideal single-stage fluorescent collectors in 20th European Photovoltaic Solar Energy Conference, Barcelona, Spain, 2005, p. 171.

${ }^{3}$ U. Rau, F. Einsele, and G. C. Glaeser, Appl. Phys. Lett. 87, 171101 (2005).

${ }^{4}$ T. Markvart, J. Appl. Phys. 99, 026101 (2006).

${ }^{5}$ U. Rau, F. Einsele, and G. C. Glaeser, Appl. Phys. Lett. 88, 176102 (2006).

${ }^{6} \mathrm{P}$. Kittidachachan, L. Danos, T. J. J. Meyer, N. Alderman, and T. Markvart, Chimia (Aarau) 61, 780 (2007).

${ }^{7}$ E. H. Kennard, Phys. Rev. 9, 58 (1917).

${ }^{8}$ G. Lasher and F. Stern, Phys. Rev. 133, A553 (1964).

${ }^{9}$ P. Würfel, J. Phys. C 15, 3967 (1982).

${ }^{10}$ C. Kittel, Thermal Physics, 2nd ed. (W. H. Freeman \& Co. Ltd., New York, 1980).

${ }^{11}$ W. Ruppel and P. Würfel, IEEE Trans. Electron Devices 27, 877 (1980).

${ }^{12}$ T. Markvart, in The Physics of Nanostructured Solar Cells, edited by V.
Badecsu and M. Paulescu (Nova Science, New York, in press).

${ }^{13}$ O. von Roos, J. Appl. Phys. 54, 1390 (1983).

${ }^{14}$ P. Laible, R. S. Knox, and T. G. Owens, J. Phys. Chem. B 102, 1641 (1998).

${ }^{15}$ R. S. Knox and L. F. Marshall, J. Lumin. 85, 209 (2000).

${ }^{16}$ D. A. Sawicki and R. S. Knox, Phys. Rev. A 54, 4837 (1996).

${ }^{17}$ Suprasil® 1, 2, 3 and Suprasil® Standard, Heraeus Quarzglas (2007).

${ }^{18}$ D. Magde, G. E. Rojas, and P. G. Seybold, Photochem. Photobiol. 70, 737 (1999).

${ }^{19}$ D. Magde, G. E. Rojas, and P. G. Seybold, Photochem. Photobiol. 75, 327 (2002).

${ }^{20}$ L. R. Wilson and B. S. Richards, PVSAT 4, Bath, England, 2008 (unpublished).

${ }^{21}$ Handbook of Chemistry and Physics, PVSAT 4 (CRC Press (Taylor \& Francis Group, Cleveland OH, 2008), Vol. 88.

${ }^{22}$ T. J. J. Meyer, J. Hlavaty, L. Smith, E. R. Freniere, and T. Markvart, in Physics and Simulation of Optoelectronic Devices XVII, edited by M. Osinski, B. Witzigmann, F. Henneberger, and Y. Arakawa, Proceedings of SPIE, Vol. 7211 (SPIE, Bellingham, WA, 2009) 72110N.

${ }^{23}$ S. J. Strickler and R. A. Berg, J. Chem. Phys. 37, 814 (1962).

${ }^{24}$ A. T. R. Williams, S. A. Winfield, and J. N. Miller, Analyst (Lond.) 108, 1067 (1983).

${ }^{25}$ W. Shockley and H. J. Quiesser, J. Appl. Phys. 32, 510 (1961). 